\title{
ON OPTIMIZATION OF FILTER BANKS WITH DENOISING APPLICATIONS
}

\author{
Sony Akkarakaran and P.P. Vaidyanathan \\ Department of Electrical Engineering 136-93 \\ California Institute of Technology \\ Pasadena, CA 91125 USA.
}

\begin{abstract}
The problem of optimization of subband coders for given input statistics has received considerable attention in recent literature. The goal in these works has been to maximize the coding gain, which is a compression performance measure under the standard quantizer noise models for high bit-rates. The optimal filter bank (FB) for this problem has been observed to be a principal component filter bank (PCFB) for the class of FB's over which the optimization is performed. The purpose of this paper is to point out a stronger connection between optimality of the FB and the principal component property, which appears to have been overlooked in the literature. We show that PCFB's are also optimal for a variety of other signal processing schemes such as noise suppression by using hard-thresholding or zeroth order Wiener filtering in the subbands.
\end{abstract}

\section{INTRODUCTION}

Suppose a filter bank (FB) is being used to analyze a signal into subbands, and then reconstruct it after some kind of processing of the subbands, as shown in Fig. 1. This paper is concerned with the problem of finding the best FB among a class $\mathcal{C}$ of uniform orthonormal $M$ channel FB's, for a particular kind of processing. We assume that the FB input $x(n)$ is modelled by realizations of a CWSS $(M)$ (wide sense cyclostationary with period $M$ ) random process (which could be wide sense stationary (WSS) in particular). To explain our usage of the term 'best FB', consider the situation where the FB is used for data compression, and so the processors $P_{i}$ in Fig. 1 are quantizers. Under the standard high bit-rate quantization noise models and assuming optimal bit allocation among the subband quantizers, minimizing the mean-square reconstruction error is equivalent to minimizing the product of the variances of the subband signals [1]. Thus, for this situation, the best FB is the one that minimizes this product of subband variances.

When the class $\mathcal{C}$ consists of all $M$-channel orthogonal transform coders, the optimum FB in $\mathcal{C}$ for the above situation is the KLT [2]. It produces subband signals $v_{i}^{(x)}(n)$ in Fig. 1 such that the vector process $\left(v_{0}^{(x)}, v_{1}^{(x)}, \ldots, v_{M-1}^{(x)}\right)^{T}$ has a diagonal autocorrelation matrix. When $\mathcal{C}$ is the class of all (unconstrained) $M$-channel orthonormal FB's, the optimum FB has been obtained in [1]. It produces a vector

Work supported in part by the National Science Foundation under Grant MIP 0703755. process $\left(v_{0}^{(x)}, v_{1}^{(x)}, \ldots, v_{M-1}^{(x)}\right)^{T}$ (see Fig. 1) that has a diagonal power-spectrum (psd) matrix, with the diagonal elements (i.e. the subband spectra) ordered according to a condition referred to as spectral majorization [1]. In both these cases, the optimum FB turns out to be a principal component filter bank (PCFB) for the class $\mathcal{C}$. PCFB's were first propounded in [3], and are defined in Section 3.

There is a stronger connection between optimality of the FB and the principal component property. This connection, which we believe is the precise reason for the optimality of PCFB's, does not seem to have been observed in the literature. The main result is that the PCFB is optimal whenever the objective to be minimized is a concave function of the subband variances produced by the FB. In the above-mentioned coding problem, the objective was the product of the subband variances. Minimizing it is equivalent to minimizing its logarithm, which is a concave function of the subband variances. Thus, the PCFB is optimal. The subsequent sections elaborate on this result, and illustrate various other $\mathrm{FB}$ based signal processing schemes for which the FB optimization involves minimizing a concave function of the subband variances. For example, this happens in the noise suppression system where the FB input $x(n)$ in Fig. 1 is a signal corrupted by zero mean additive white noise, and the processors $P_{i}$ are either zeroth order Wiener filters or hard-thresholders. Thus a PCFB is optimal for all these schemes as well.

\section{PROBLEM FORMULATION}

We are given a class $\mathcal{C}$ of $M$-channel orthonormal FB's, and a set of $M$ subband processors $P_{i}, i=0,1, \ldots, M-1$ (numbered arbitrarily). A processor is simply a well-defined function that maps input sequences to output sequences. The specification of this function might be independent of any statistical properties that the input sequences are assumed to have; or on the other hand it might not. Examples of the former kind of processors are fixed LTI systems and memoryless squaring devices. Examples of the latter kind are Wiener filters and pdf-optimized quantizers. The signal processing system consists of a FB from $\mathcal{C}$ and the processors $P_{i}$ used in its subbands as shown in Fig. 1.

For this system, we define the subband variance vector as $\mathbf{v}=\left(\sigma_{0}^{2}, \sigma_{1}^{2}, \ldots, \sigma_{M-1}^{2}\right)^{T}$ whose $i$-th entry is the variance of the subband signal input to the processor $P_{i}$, for $i=0,1, \ldots, M-1$. It can be computed for each FB given the psd matrix of the $M$-fold blocked version of the scalar process $x(n)$ input to the FB. The optimization search 
space is defined as the set $S$ consisting of all subband variance vectors associated with all FB's in the given class $\mathcal{C}$. We do not assume any constraint as to which processor $P_{i}$ to use in which subband of the FB. The set $S$ is therefore 'permutation-symmetric': If $\mathbf{v}$ is in $S$ then all vectors obtained from $\mathbf{v}$ by permuting its entries are also in $S$. The problem at hand is to find the $\mathrm{FB}$ from $\mathcal{C}$ that minimizes an objective function that is well-defined on the class $\mathcal{C}$. The assumption we make on this objective is that it can be fully evaluated at each $\mathrm{FB}$ in $\mathcal{C}$ given the variances of the subband signals that the FB produces, and the information as to which variance enters which processor $P_{i}$. Thus the objective can be represented by a real-valued function $g$ defined on the search space $S$. This happens for a number of FB based signal processing schemes, as will be seen later.

Notice that the objective need not be symmetric in its arguments, i.e. $g$ could have different values at two different vectors in $S$ which are permutations of each other. This usually happens because the subband processors $P_{i}$ are not identical. To find the best FB, we find the vector $\mathbf{v}_{\text {opt }} \in S$ that minimizes $g$ over $S$. The optimum FB is then identified as any $\mathrm{FB}$ in $\mathcal{C}$ whose subband variances are the entries in $\mathbf{v}_{\text {opt }}$, provided the subbands of this FB are coupled to the subband processors in the order corresponding to $\mathbf{v}_{\text {opt }}$.

\section{PCFB'S AND THEIR OPTIMALITY}

\subsection{Definitions and statement of result}

Majorization : Given two sets $A, B$ each having $M$ real numbers (not necessarily distinct), $A$ is defined to majorize $B$ if the elements $a_{i} \in A$ and $b_{i} \in B$ arranged in descending order $a_{0} \geq a_{1} \geq \ldots \geq a_{M-1}$, and $b_{0} \geq b_{1} \geq$ $\ldots \geq b_{M-1}$, obey the property that

$$
\sum_{i=0}^{P} a_{i} \geq \sum_{i=0}^{P} b_{i} \text { for all } P=0,1, \ldots, M-1,
$$

with equality holding when $P=M-1$.

PCFB's : Let us be given a class $\mathcal{C}$ of uniform orthonormal $M$-channel FB's, and the power-spectrum of the input to the FB. A PCFB for the class $\mathcal{C}$ is defined to be a FB in $\mathcal{C}$ whose set of subband variances majorizes the set of subband variances of any $\mathrm{FB}$ in $\mathcal{C}$. Alternatively, a PCFB may be defined as a FB that minimizes (over all FB's in $\mathcal{C}$ ) the mean-square error caused by dropping the $P$ weakest (lowest variance) subbands, for any $P=0,1, \ldots, M$. The equivalence of these two definitions is due to the fact that dropping subbands results in a mean-square reconstruction error that is the sum of the variances of the dropped subband signals (upto a constant scale-factor of $\frac{1}{M}$ ). The PCFB and its existence depends on both the class $\mathcal{C}$ and the input spectrum.

Main result on PCFB optimality. Let $\mathcal{C}$ be a perfectly arbitrary class of uniform $M$-channel orthonormal FB's, such that a PCFB exists for this class. Then the search space $S$ has the property that its convex hull $\operatorname{co}(S)$ is a polytope (defined in Section 3.2 below). All the corners of this polytope are permutations of each other, and are elements of $S$ that correspond to the PCFB. The objective $g$ to be minimized is a real-valued function on $S$. If it has an extension to $\operatorname{co}(S)$ on which it is concave, then at least one of the corners of the polytope is a minimum of $g$. Thus, the PCFB is always optimal. Further if $g$ is strictly concave, then its minimum is necessarily at some corner of the polytope, i.e. the optimum FB is necessarily a PCFB.

\subsection{Discussion of the result}

Recall that a function $f: D \rightarrow \mathcal{R}$ is defined to be concave if given any $\mathbf{x}, \mathbf{y} \in D$ and $\mu \in[0,1]$,

$$
f(\mu \mathbf{x}+(1-\mu) \mathbf{y}) \geq \mu f(\mathbf{x})+(1-\mu) f(\mathbf{y})
$$

Graphically, this means that the function is always above its chord, as is seen from the examples in Fig. 2. Here the domain $D$ of $f$ is some subset of $\mathcal{R}^{M}$, however the definition makes sense only if $D$ is a convex set. $D$ is defined to be convex if any convex combination of any finite set of elements from $D$ is also in $D$. A convex combination of the vectors $\mathbf{x}_{i}, i=1,2, \ldots, N$ is a vector of the form $\sum_{i=1}^{N} \alpha_{i} \mathbf{x}_{i}$ for some $\alpha_{i} \in[0,1]$ that satisfy $\sum_{i=1}^{N} \alpha_{i}=1$. The convex hull of a set $E$ is defined as the set of all possible convex combinations using vectors from $E$, and is denoted by $\operatorname{co}(E)$. A convex polytope is defined as the convex hull of a finite set of points. Given such a polytope $\operatorname{co}(E)$, we can assume that no element of $E$ is a convex combination of other elements of $E$. This is because any such element can be deleted from $E$ without changing $\operatorname{co}(E)$. Under this condition, the elements of the finite set $E$ are called corners of the polytope. The reason for these names is clear from examples of polytopes embedded in 1,2 or 3 - dimensional space as shown in Fig. 3.

Now if the function $f: D \rightarrow \mathcal{R}$ is concave and $D$ is a polytope, then at least one of the corners of $D$ is a minimum of $f$ over $D$. This fact is illustrated in Fig. 4, which makes it intuitively clear. Indeed it is a standard result in convex function theory, provable directly from the definitions of polytopes and concave functions.

In our problem, $f=g$, the objective function; and $D=\operatorname{co}(S)$ where $S$ is the optimization search-space (defined in Section 2). Further, if a PCFB exists then it can be shown that $\operatorname{co}(S)$ is a polytope whose corners correspond to the PCFB. This proves the main result on PCFB optimality (Section 3.1). The crucial fact that $\operatorname{co}(S)$ is a polytope when a PCFB exists, follows from the geometrical meaning of majorization [4]. It is proved in detail in [5]. Thus, when a PCFB exists, the analytical tractability of the FB optimization problems can be attributed to this special structure of the search-space $S$. The situation when a PCFB does not exist is discussed in $[6,5]$. Extensions to nonuniform FB's are discussed in [5].

\section{PROBLEMS WITH CONCAVE OBJECTIVES}

This section shows a number of filter-bank based signal processing schemes for which the FB optimization objective is a concave function of the subband variances of the FB. Thus, from Section 3, if a PCFB exists then it is optimal for all these schemes. 


\subsection{General features and structure of the problems}

Consider the generic FB based signal processing scheme shown in Fig. 1. We denote by $v_{i}^{(s)}(n)$ the $i$-th subband signal generated by feeding the signal $s(n)$ as input to the FB, for $i=0,1, \ldots, M-1$ (where the subbands are numbered according to the subband processors they are associated with). The system of Fig. 1 is aimed at producing a certain desired signal $d(n)$ at the FB output. It is deemed to be optimized if the actual FB output $y(n)$ is 'as close to' $d(n)$ as possible, i.e. some measure of the error signal $e(n)=d(n)-y(n)$ is minimized. To formulate this measurc, wc assumc that the signals $x(n)$ and $d(n)$ are jointly CWSS $(M)$. Often the subband processors $P_{i}$ are such that the error $e(n)$ is also a CWSS $(M)$ process - this happens whenever the $P_{i}$ are LTI systems for instance. The error measure is then the variance of the process $e(n)$ averaged over the period of cyclostationarity $M$. If the FB is orthonormal, this measure takes the form

$$
\begin{gathered}
\frac{1}{M} \sum_{i=0}^{M-1} E\left[\left|v_{i}^{(e)}\right|^{2}\right], \text { where } \\
v_{i}^{(e)}(n)=v_{i}^{(d)}(n)-v_{i}^{(y)}(n), \text { for } i=0,1, \ldots, M-1
\end{gathered}
$$

Thus $v_{i}^{(d)}(n)$ serves as the desired response that the processor $P_{i}$ must try to approximate at its output as best as possible in the sense of minimizing $E\left[\left|v_{i}^{(e)}\right|^{2}\right]$.

Let the variance of $v_{i}^{(x)}(n)$ be denoted by $\sigma_{i}^{2}$. The subband variance vector (defined in Section 2) is thus $\mathbf{v}=$ $\left(\sigma_{0}^{2}, \sigma_{1}^{2}, \ldots, \sigma_{M-1}^{2}\right)^{T}$. In many situations, the processors $P_{i}$ are such that

$$
E\left[\left|v_{i}^{(e)}(n)\right|^{2}\right]=h_{i}\left(\sigma_{i}^{2}\right)
$$

where $h_{i}$ is some function that depends on the kind of processor $P_{i}$, and is independent of the FB. Thus, for such processors $P_{i},(3)$ and (5) show that the FB optimization objective $g$ takes the form

$$
g(\mathbf{v})=\frac{1}{M} \sum_{i=0}^{M-1} h_{i}\left(\sigma_{i}^{2}\right)
$$

If the $h_{i}$ are concave on $[0, \infty)$ then $g$ is concave on $\operatorname{co}(S)$ where. $S$ is the search space (defined in Section 2). Thus, from Section 3, PCFB's are optimal whenever the $h_{i}$ are concave on $[0, \infty)$. We may note that often all the $h_{i}$ are identical functions, the typical reason being that the processors $P_{i}$ are identical. In this case $g$ is symmetric in its arguments, i.e. it is not changed by permutations of the $\sigma_{i}^{2}$. Hence the subbands of optimum FB can be coupled to the subband processors in an arbitrary fashion. If the $h_{i}$ are not identical, $g$ loses this symmetry property, and then the coupling has to be done in a definite way to ensure optimality. In the high bit-rate coding problem with optimal bit allocation [1], $h_{i}(x)=\log (x)$. At low bit-rates, let the $i$-th quantizer have a normalized quantizer function $f_{i}$. Under the assumption that $f_{i}$ is independent of the FB (thus ruling out pdf-optimized quantizers), $h_{i}(x)=f_{i}\left(b_{i}\right) x$ [7] where $b_{i}$ is the number of bits alloted to the $i$-th subband. Since all these $h_{i}$ are concave (on $[0, \infty)$ ), this gives a direct proof of the results of $[1,7]$. Further details regarding optimal bit allocation can be found in [5].

\subsection{Denoising/Wiener filtering for white noise}

Here the FB input in Fig. 1 is $x(n)=s(n)+\mu(n)$ where $s(n)$ is a pure signal and $\mu(n)$ is zero mean white noise. We assume that $\mu(n)$ is uncorrelated to $s(n)$, and has a fixed known variance $\eta^{2}>0$. The overall desired output signal is $d(n)=s(n)$. The $i$-th subband process $v_{i}^{(x)}(n)$ contains a signal component $v_{i}^{(s)}(n)$ and a zero mean additive noise component $v_{i}^{(\mu)}(n)$. Orthonormality of the FB ensures that the subband noise components are also white with variance $\eta^{2}$, and are uncorrelated to the signal components.

\subsubsection{Subband processors as constant multipliers}

Suppose each processor $P_{i}$ is a fixed multiplier of value $k_{i}$ (memoryless LTI system). Then

$$
v_{i}^{(e)}(n)=v_{i}^{(d)}(n)-v_{i}^{(y)}(n)=\left(1-k_{i}\right) v_{i}^{(s)}(n)-k_{i} v_{i}^{(\mu)}(n)
$$

Thus, since $v_{i}^{(\mu)}(n)$ is zero mean and uncorrelated to $v_{i}^{(s)}(n)$,

$$
E\left[\left|v_{i}^{(e)}(n)\right|^{2}\right]=\left|1-k_{i}\right|^{2} \sigma_{i}^{2}+\left|k_{i}\right|^{2} \eta^{2}
$$

where $\sigma_{i}^{2}$ is the $i$-th subband variance corresponding to the signal $s(n)$, i.e. $\sigma_{i}^{2}=E\left[\left|v_{i}^{(s)}(n)\right|^{2}\right]$. Comparison with (5) identifies the $h_{i}$ in $(5,6)$ as

$$
h_{i}(x)=\left|1-k_{i}\right|^{2} x+\left|k_{i}\right|^{2} \eta^{2}
$$

which is linear in $x$, and is hence concave. Notice that while in (5), $\sigma_{i}^{2}$ was the variance of the subband signal $v_{i}^{(x)}(n)$ corresponding to the FB input $x(n)$, here it is the variance of $v_{i}^{(s)}(n)=v_{i}^{(x)}(n)-v_{i}^{(\mu)}(n)$. This distinction is not very serious here: It says that the optimal FB is a PCFB for the signal $s(n)$ (as opposed to the FB input $x(n)$ ). However in the present problem, because the noise is white, and $E\left[\left|v_{i}^{(s)}(n)\right|^{2}\right]=\sigma_{i}^{2}=E\left[\left|v_{i}^{(x)}(n)\right|^{2}\right]-\eta^{2}$, we find that PCFB's for $s(n)$ are also PCFB's for $x(n)$ and vice verca. The situation when the noise is colored is more involved [5]: In certain cases it is possible to show optimality of a simultaneous PCFB for signal and noise (if it exists).

\subsubsection{Using multipliers matched to input statistics.}

If the processor $P_{i}$ is a zeroth order Wiener filter, then it is a multiplier given by

$$
k_{i}=\frac{\sigma_{i}^{2}}{\sigma_{i}^{2}+\eta^{2}}
$$

where $\sigma_{i}^{2}$ is the variance of $v_{i}^{(s)}(n)$. On the other hand, if $P_{i}$ is a hard-threshold operator, it keeps or kills the subband depending on whether the variance of the subband signal component is greater than or less than the variance of the noise component. In this case, it is a multiplier given by

$$
k_{i}= \begin{cases}1 & \text { if } \sigma_{i}^{2} \geq \eta^{2} \\ 0 & \text { otherwise }\end{cases}
$$

These schemes can be implemented in practice by estimating $\sigma_{i}^{2}$ from the subband process $v_{i}^{(x)}(n)$, which is possible 
since $\eta^{2}$ is known. Substituting these $k_{i}$ in (8) and comparing with (5) shows that we have a new set of $h_{i}$, i.e.

$$
h_{i}(x)=\left\{\begin{array}{cc}
\frac{x \eta^{2}}{x+\eta^{2}} & \text { if } P_{i}=0^{\text {th }} \text { order Wiener filter } \\
\min \left(x, \eta^{2}\right) & \text { if } P_{i}=\text { hard thresholder }
\end{array}\right.
$$

These functions are plotted in Fig. 5, and are concave on $[0, \infty)$. Thus the PCFB is optimal for any mixture of zeroth order Wiener filters and hard thresholders in the subbands.

Notice that in Fig. 5, the Wiener filter curve lies fully below the hard threshold curve, i.e. the Wiener filter yields a lower mean-square error. This is expected since it is by definition the optimum choice of multiplier $k_{i}$ in this sense. Use of hard thresholds is motivated by other considerations $[8,9]$, for example to effect a bias-variance tradeoff. Indeed, (7) shows that when $s(n)$ has nonzero mean and $k_{i} \in[0,1]$, the estimation bias decreases if $k_{i}$ increases. The Wiener filter always produces bias, while the hard thresholder produces zero bias whenever it results in $k_{i}=1$.

\section{CONCLUDING REMARKS}

We have pointed out a basic connection between FB optimization and the principal component property. We have shown that PCFB's are optimal for various signal processing schemes such as subband denoising using zeroth order Wiener filters and hard thresholders. A companion paper [6] discusses these optimization problems in situations where a PCFB does not exist. Extensions to colored noise suppression, and to nonuniform FB's, can be found in [5].

\section{REFERENCES}

[1] P.P.Vaidyanathan, "Theory of Optimal Orthonormal Subband Coders," IEEE Trans. SP, vol. 46, no. 6, pp. 1528-1543, June 1998.

[2] Y.Huang and P.M.Schultheiss, "Block quantization of correlated Gaussian random variables," IEEE Trans. Commun. Syst., vol. C-10, pp. 289-296, Sept. 1963.

[3] M.K.Tsatsanis and G.B.Giannakis, "Principal Component Filter Banks for Optimal Multiresolution Analysis," IEEE Trans. SP, pp. 1766-1777, Aug. 1995.

[4] R.A.Horn and C.R.Johnson, Matrix Analysis. Cambridge University Press, 1985.

[5] S.Akkarakaran and P.P.Vaidyanathan, "General Results on Filter Bank Optimization with Convex Objectives, and the Optimality of Principal Component Filter Banks," in preparation.

[6] S.Akkarakaran and P.P.Vaidyanathan, "The best basis problem, compaction problem and PCFB design problems," in Proc. IEEE ISCAS, 1999.

[7] A.Kirac and P.P.Vaidyanathan, "Optimality of Orthonormal Transforms for Subband Coding," in Proc. IEEE DSP Workshop, Utah, August 1998.

[8] D.L.Donoho and I.M.Johnstone, "Ideal spatial adaptation by wavelet shrinkage," Biometrika, vol. 81 , no. 3, pp. 425-455, 1994.

[9] S.P.Ghael, A.M.Sayeed, and R.G.Baraniuk, "Improved Wavelet Denoising via Empirical Wiener Filtering," in Proc. SPIE, San Diego, July 1997, pp. 389-399.

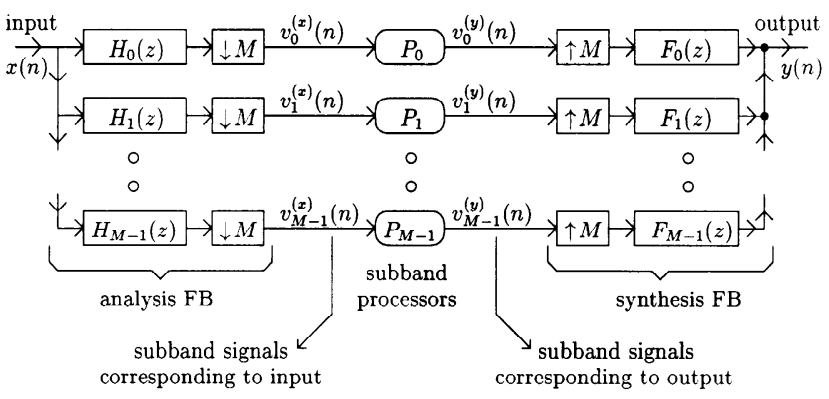

Figure 1: Generic FB based signal processing scheme.
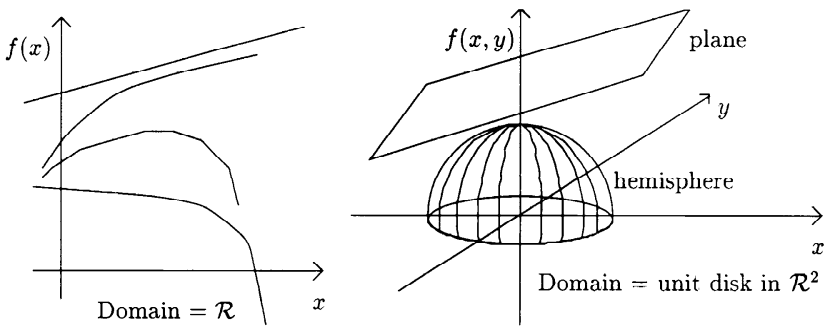

Figure 2: Concave functions on different domains.

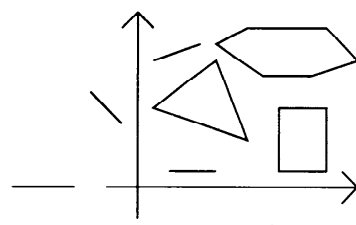

In $\mathcal{R}$
In $\mathcal{R}^{2}$

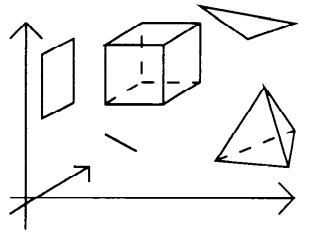

In $\mathcal{R}^{3}$
Figure 3: Polytopes in $\mathcal{R}, \mathcal{R}^{2}$ and $\mathcal{R}^{3}$.
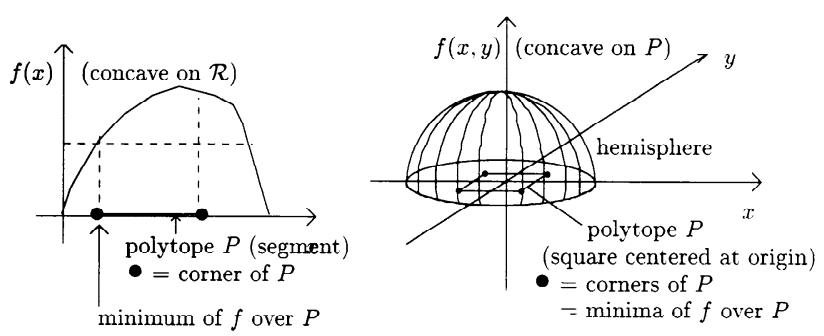

Figure 4: Optimality of corners of polytopes.

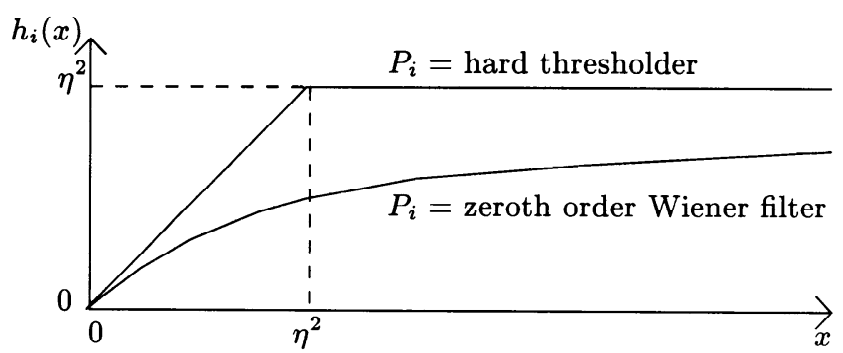

Figure 5: Subband error functions. 